\title{
Effect of space charge on linear coupling and gradient errors in high-intensity rings
}

\author{
M. Aslaninejad* \\ Department of Physics, Amir Kabir University and Institute for Studies in Theoretical Physics and Mathematics (IPM), \\ Tehran, Iran \\ I. Hofmann \\ Gesellschaft für Schwerionenforschung, Planckstrasse 1, 64291 Darmstadt, Germany
}

(Received 22 October 2003; published 24 December 2003)

\begin{abstract}
We study the response of coasting beams in the presence of space charge to linear coupling as well as gradient errors, based on linearizing the self-consistent Vlasov-Poisson equation. We determine the coherent shift of the resonance conditions as well as the response on lattice errors in smooth approximation, but allowing for arbitrary tune and/or emittance ratios. A characteristic feature is cancellation between the external forces due to the lattice errors and the beam induced space-charge forces, if the resonance condition is satisfied for the (space-charge shifted) single-particle tunes. For the linear coupling mode and small tune split we also identify a parameter region, where spontaneous instability exists in addition to the resonance.
\end{abstract}

DOI: 10.1103/PhysRevSTAB.6.124202

PACS numbers: 41.75.-i, 29.20.-c

\section{INTRODUCTION}

In designing circular accelerators for high-intensity beams one has to take into account the combined effect of space charge and lattice imperfections. The issue of envelope resonances and instabilities has received new attention in several recent papers (see Refs. [1-3]), whereas the effect of space charge on linear coupling due to skew quadrupoles - the main topic of this work- has hardly been addressed. Knowledge of the precise resonance condition for linear coupling is, on the other hand, crucial in a high-intensity ring where coupling is used to exchange or equalize emittances.

A common feature of all resonances in the presence of space charge is that the resonance condition $m \nu_{0, x}+$ $n \nu_{0, y}=N$ cannot be replaced simply by using the space-charge shifted incoherent betatron frequencies $\nu_{x}, \nu_{y}$, since the full ensemble of particles may respond to the resonance in a coherent way. For such a coherently oscillating beam the resonance condition is shifted and should be replaced by the coherent resonance condition $m \nu_{x}+n \nu_{y}+\Delta \Omega=N$. This condition expresses the fact that the coherent density mode resonates with the error harmonic $N$. Note that in this context "coherent oscillation" is not relating to a beam displacement as a whole (dipole mode), but to an oscillation of the density profile. In this paper we use the linearized Vlasov-Poisson equation in 2D coasting beam approximation and study the response of an anisotropic Kapchinskij-Vladimirskij (KV) distribution beam exposed to second order lattice errors. Our analysis extends the previous work of one of the authors (see Ref. [4]) on the transverse eigenmodes in an ideal lattice to the case of nonideal lattices with errors.

*Email address: m.aslaninejad@gsi.de
It also extends the work of Venturini and Gluckstern [1], who have studied isotropic beams in symmetric focusing with gradient errors, to the case of anisotropic beams with both different tunes and emittances, and including linear coupling. Throughout the paper we employ KV emittances (four times the rms emittances) and beam sizes of uniform beams (twice the rms size). Our results may be applied to waterbag or Gaussian distributions using rms-equivalent beams, which requires careful checking by future simulations.

The structure of the paper is as follows: we start in Sec. II with an equilibrium phase-space distribution and derive the linearized Vlasov-Poisson equations in the presence of lattice errors. A brief review of the solution of this equation in the absence of lattice errors is given in Sec. IV. Next we derive the resonance condition and a relation for the expected tune shifts for the second order even modes (envelope modes) as well as for the linear coupling (second order odd or skew) modes. We discuss the cancellation effects between lattice errors and spacecharge forces in Sec. IVC. In Appendix A we compare the obtained tune shift results for the second order even modes with previous results, and in Appendix B we use the envelope equations to further illustrate the force cancellation principle.

\section{BASIC EQUATIONS}

We choose a Kapchinskij-Vladimirskij equilibrium distribution function with uniform density profile in configuration space

$$
n_{0, b}(x, y)= \begin{cases}n_{0, b}=\mathrm{const}, & \text { if } \frac{x^{2}}{a^{2}}+\frac{y^{2}}{b^{2}} \leq 1, \\ 0, & \text { if } \frac{x^{2}}{a^{2}}+\frac{y^{2}}{b^{2}}>1,\end{cases}
$$

and $a$ and $b$ the semiaxes of the confining ellipse. Here, it 
is assumed that the transverse beam dimensions are small in comparison with the radius of the beam pipe so that we can ignore image charges. From Poisson's equation we readily obtain the effective potential inside the beam valid for a uniform distribution of particles in free space with charge $q$ and line density $\tilde{N}=n_{0, b} \pi a b$ (transformed to the laboratory frame):

$$
\psi_{\mathrm{eff}}=\left[\frac{q \tilde{N}}{2 \epsilon_{0} \pi \gamma^{2} a(a+b)} x^{2}+\frac{q \tilde{N}}{2 \epsilon_{0} \pi \gamma^{2} b(a+b)} y^{2}\right]
$$

We now assume linear and continuous external focusing forces for the equilibrium beam, and define betatron frequencies in the absence of space charge $\nu_{0, x}$ and $\nu_{0, y}$, corresponding to the oscillation frequencies of a single particle under the applied focusing field in the $x$ and $y$ directions. Considering in addition the self-field effects with their defocusing contribution, we can write the full Hamiltonian as

$$
\begin{aligned}
H_{0}\left(p_{x}, p_{y}, x, y\right)= & \frac{p_{x}^{2}+m^{2} \gamma^{2} \nu_{0, x}^{2} x^{2}}{2 m \gamma}+\frac{p_{y}^{2}+m^{2} \gamma^{2} \nu_{0, y}^{2} y^{2}}{2 m \gamma} \\
& -q \psi_{\mathrm{eff}},
\end{aligned}
$$

where $m$ is the particle rest mass. With $\omega_{p}^{2}=$ $q^{2} \tilde{N} / \epsilon_{0} \pi m \gamma^{3} a b$ the beam plasma frequency in the laboratory frame, one can write the actual particle oscillation frequencies $\nu_{x}, \nu_{y}$ in the presence of space charge as

$$
\begin{aligned}
& \nu_{x}^{2}=\nu_{0, x}^{2}-\omega_{p}^{2} /(1+a / b), \\
& \nu_{y}^{2}=\nu_{0, y}^{2}-\omega_{p}^{2} /(1+b / a) .
\end{aligned}
$$

The full Hamiltonian can now be divided into two separate parts for the $x$ and $y$ directions:

$$
H_{0, x}=\frac{p_{x}^{2}+m^{2} \gamma^{2} \nu_{x}^{2} x^{2}}{2 m \gamma}, \quad H_{0, y}=\frac{p_{y}^{2}+m^{2} \gamma^{2} \nu_{y}^{2} y^{2}}{2 m \gamma} .
$$

The transverse energy anisotropy $T$, hence the ratio of oscillation energies in $x$ and $y$ directions, can be written in terms of betatron tunes and emittances as $T=$ $a^{2} \nu_{x}^{2} / b^{2} \nu_{y}^{2}=\epsilon_{x} \nu_{x} / \epsilon_{y} \nu_{y}$. The anisotropic KV distribution can now be expressed in terms of a $\delta$ function of the linear combination of the two separate Hamiltonians [4]

$$
f_{0}\left(x, y, p_{x}, p_{y}\right)=\frac{\tilde{N} T \nu_{y} / \nu_{x}}{2 \pi^{2} m \gamma a^{2}} \delta\left(H_{0, x}+T H_{0, y}-\frac{1}{2} m \gamma \nu_{x}^{2} a^{2}\right),
$$

which is consistent with a uniform density within the boundary of the confining ellipse.

We now introduce a lattice error described by a $z^{-}$component of an "error" vector potential $\vec{A}^{e}$. Considering small-amplitude perturbations about $f_{0}$, $\vec{E}_{0}, \vec{B}_{0}$, and expressing

$$
\begin{aligned}
f & =f_{0}\left(H_{0, x}+H_{0, y}\right)+f_{1}\left(x, y, p_{x}, p_{y}, t\right), \\
\vec{E} & =\vec{E}_{0}(x, y)+\vec{E}_{1}(x, y, t), \\
B & =\vec{B}_{0}(x, y)+\vec{B}_{1}(x, y, t),
\end{aligned}
$$

we linearize Vlasov's equation and keep only first-order terms in $f_{1}, \vec{E}_{1}, \vec{B}_{1}$ to obtain (see, for example, Ref. [5])

$$
\left[\frac{\partial}{\partial t}+\overrightarrow{\boldsymbol{v}} \frac{\partial}{\partial \vec{x}}+q\left(\vec{E}_{0}+\overrightarrow{\boldsymbol{v}} \times \vec{B}_{0}\right) \frac{\partial}{\partial \vec{p}}\right] f_{1}=-q\left(\vec{E}_{1}+\overrightarrow{\boldsymbol{v}} \times \vec{B}_{1}\right) \frac{\partial}{\partial \vec{p}} f_{0} .
$$

A general remark may be appropriate here. In our approach the equilibrium is a beam matched to the ideal lattice. Turning on errors instantaneously results in timedependent perturbations of this equilibrium. An alternative approach would be to search for a matched beam in the presence of a gradient or skew error. Plugging into such a system the original matched solution (of the ideal lattice) would then result in oscillations about the errormatched beam - another way of looking at the perturbations considered here.

With $\vec{E}_{1}=-\nabla \Phi$ and $\vec{B}_{1}=\nabla \times \vec{A}^{e}$, we find

$$
\begin{aligned}
\frac{d f_{1}}{d t} \equiv \frac{\partial f_{1}}{\partial t}+\frac{p_{x}}{m \gamma} \frac{\partial f_{1}}{\partial x}+\frac{p_{y}}{m \gamma} \frac{\partial f_{1}}{\partial y}-m \gamma \nu_{x}^{2} x \frac{\partial f_{1}}{\partial p_{x}}-m \gamma \nu_{y}^{2} y \frac{\partial f_{1}}{\partial p_{y}} \\
=\frac{\tilde{N} T q \nu_{y} / \nu_{x}}{2 \pi^{2} m^{2} \gamma^{4} a^{2}}\left[p_{x} \frac{\partial \Phi}{\partial x}+T p_{y} \frac{\partial \Phi}{\partial y}\right. \\
\left.\quad-v_{z} \gamma^{2}\left(p_{x} \frac{\partial A_{z}^{e}}{\partial x}+T p_{y} \frac{\partial A_{z}^{e}}{\partial y}\right)\right] \delta^{\prime}\left[\frac{p_{x}^{2}+m^{2} \gamma^{2} \nu_{x}^{2}}{2 m \gamma}+T \frac{p_{y}^{2}+m^{2} \gamma^{2} \nu_{y}^{2} y^{2}}{2 m \gamma}-\frac{1}{2} m \gamma \nu_{x}^{2} a^{2}\right] .
\end{aligned}
$$

The perturbed electrostatic potential $\Phi$ is in turn selfconsistently obtained from the linearized Poisson equation

$$
\nabla^{2} \Phi=-\frac{q}{\epsilon_{0}} n_{1}(x, y)=-\frac{q}{\epsilon_{0}} \int f_{1} d p_{x} d p_{y}
$$

Equations (8) and (9) form a closed set that can be solved with an appropriate boundary condition.

\section{INTEGRATION OF VLASOV'S EQUATION}

We follow the technique used in Ref. [4] and integrate Eq. (8) along the orbits of the unperturbed Hamiltonian by introducing a phase angle $\phi \equiv \nu_{x} t$ such that at time $t^{*}=t$, hence at $\phi^{*}=\phi$, the orbit passes through the point $\left(x, y, p_{x}, p_{y}\right)$ in phase space. Using the above Hamiltonian, the corresponding single-particle equations 
of motion read

$$
\begin{array}{ll}
\dot{p}_{x}=-m \gamma \nu_{x}^{2} x, & \dot{x}=p_{x} / m \gamma, \\
\dot{p}_{y}=-m \gamma \nu_{y}^{2} y, & \dot{y}=p_{y} / m \gamma .
\end{array}
$$

One then obtains

$$
\begin{aligned}
x^{*}\left(t^{*}\right) & =\frac{p_{x}}{m \gamma \nu_{x}} \sin \left(\phi^{*}-\phi\right)+x \cos \left(\phi^{*}-\phi\right), \\
p_{x}^{*}\left(t^{*}\right) & =p_{x} \cos \left(\phi^{*}-\phi\right)-m \gamma x \nu_{x} \sin \left(\phi^{*}-\phi\right), \\
y^{*}\left(t^{*}\right) & =\frac{p_{y}}{m \gamma \nu_{y}} \sin \left[\alpha\left(\phi^{*}-\phi\right)\right]+y \cos \left[\alpha\left(\phi^{*}-\phi\right)\right], \\
p_{y}^{*}\left(t^{*}\right) & =p_{y} \cos \left[\alpha\left(\phi^{*}-\phi\right)\right]-m \gamma y \nu_{y} \sin \left[\alpha\left(\phi^{*}-\phi\right)\right] .
\end{aligned}
$$

Here we have introduced the ratio of the betatron frequencies $\alpha \equiv \nu_{y} / \nu_{x}$. Assuming that $\alpha$ is rational $-\alpha=$ $n / j$ with $n$ and $j$ some integer numbers - the orbit given by Eqs. (11) is exactly periodic in $\phi^{*}$ with period $L=$ $2 \pi j$. The perturbed distribution function along the unperturbed orbit, $f_{1}(t, \phi)$, is then also periodic in $\phi$, and the total derivative in Eq. (8) can be written in terms of two variables only,

$$
\frac{d f_{1}}{d t}=\frac{\partial f_{1}}{\partial t}+\nu_{x} \frac{\partial f_{1}}{\partial \phi}
$$

We can now assume an explicit time dependence for a single eigenmode by introducing a coherent mode frequency $\omega, f_{1}=f_{1}(\phi) e^{-i \omega t}, \Phi=\Phi(\phi) e^{-i \omega t}$, so that $f_{1}$ can be determined by integrating the total derivative $d f_{1} / d t$ over a full period $L$ of the unperturbed orbit:

$$
\int_{\phi}^{\phi+L} \frac{d f_{1}}{d t^{*}} d \phi^{*}=\nu_{x} f_{1}(\phi) e^{-i \omega \phi / \nu_{x}}\left(e^{-i \omega L / \nu_{x}}-1\right)
$$

Hence, by inserting Eq. (8) into Eq. (13), introducing $u \equiv$ $\phi^{*}-\phi$, and dropping the explicit time dependence, we obtain

$$
\begin{aligned}
f_{1}(\phi)= & \frac{\tilde{N} T q \nu_{y} / \nu_{x}^{2}}{2 \pi^{2} m^{2} \gamma^{4} a^{2}}\left(e^{-i \omega L / \nu_{x}}-1\right)^{-1} \delta^{\prime}\left[\frac{p_{x}^{2}+m^{2} \gamma^{2} \nu_{x}^{2}}{2 m \gamma}+T \frac{p_{y}^{2}+m^{2} \gamma^{2} \nu_{y}^{2} y^{2}}{2 m \gamma}-\frac{1}{2} m \gamma \nu_{x}^{2} a^{2}\right] \\
& \times \int_{0}^{L}\left[\left(p_{x}^{*} \frac{\partial \Phi}{\partial x^{*}}+T p_{y}^{*} \frac{\partial \Phi}{\partial y^{*}}\right)-v_{z} \gamma^{2}\left(p_{x}^{*} \frac{\partial A_{z}^{e}}{\partial x^{*}}+T p_{y}^{*} \frac{\partial A_{z}^{e}}{\partial y^{*}}\right)\right] e^{-i\left(\omega / \nu_{x}\right) u} d u,
\end{aligned}
$$

with $x^{*}$, etc., according to Eqs. (11). We now substitute $f_{1}$ of Eq. (14) into Eq. (9), and carry out the momentum space integration by introducing polar coordinates $P$ and $\Theta$, so that $p_{x}=P \cos \Theta$ and $T^{1 / 2} p_{y}=P \sin \Theta$. Partial integration over $P^{2}$ leads to both a nonvanishing boundary term for $P^{2}=0$ describing a surface charge perturbation on the unperturbed beam boundary, and a volume part of the charge perturbation

$$
\begin{aligned}
\nabla^{2} \Phi=\frac{\tilde{N} \sqrt{T} q^{2} \nu_{y} / \nu_{x}^{2}}{2 \pi^{2} \epsilon_{0} m \gamma^{3} a^{2}}\left(e^{-2 \pi i j\left(\omega / \nu_{x}\right)}-1\right)^{-1}\{ & 2 \pi \delta\left[\frac{1}{2} m \gamma\left(\nu_{x}^{2} x^{2}+T \nu_{y}^{2} y^{2}-\nu_{x}^{2} a^{2}\right)\right] \int_{0}^{2 \pi j} e^{-i\left(\omega / \nu_{x}\right) u}\left[\left(p_{x}^{*} \frac{\partial \Phi}{\partial x^{*}}+T p_{y}^{*} \frac{\partial \Phi}{\partial y^{*}}\right)\right. \\
& \left.-v_{z} \gamma^{2}\left(p_{x}^{*} \frac{\partial A_{z}^{e}}{\partial x^{*}}+T p_{y}^{*} \frac{\partial A_{z}^{e}}{\partial y^{*}}\right)\right] P_{P^{2}=0} d u+2 m \gamma \int_{0}^{2 \pi j} e^{-i\left(\omega / \nu_{x}\right) u} \frac{d}{d P^{2}} \\
& \left.\times\left[\left(p_{x}^{*} \frac{\partial \Phi}{\partial x^{*}}+T p_{y}^{*} \frac{\partial \Phi}{\partial y^{*}}\right)-v_{z} \gamma^{2}\left(p_{x}^{*} \frac{\partial A_{z}^{e}}{\partial x^{*}}+T p_{y}^{*} \frac{\partial A_{z}^{e}}{\partial y^{*}}\right)\right]_{P^{2}=m^{2} \gamma^{2}\left(\nu_{x}^{2}\left(a^{2}-x^{2}\right)-T \nu_{y}^{2} y^{2}\right)} d u d \Theta\right\} .
\end{aligned}
$$

The unknown solutions for $\Phi$ can be regarded as finite order polynomials in $x$ and $y$ in the interior of the beam that are matched to outside solutions satisfying Laplaces's equation in elliptic coordinates

$$
\nabla^{2} \Phi=\frac{1}{c^{2}\left(\cosh ^{2} \xi-\cos ^{2} \varphi\right)}\left(\frac{\partial^{2} \Phi}{\partial \xi^{2}}+\frac{\partial^{2} \Phi}{\partial \varphi^{2}}\right)=0, \quad x=c \cosh \xi \cos \varphi, \quad y=c \sinh \xi \sin \varphi, \quad c^{2}=a^{2}-b^{2} .
$$

Here we assume without loss of generality that $a \geq b$. The outside solution $\left(\xi>\xi_{0}\right.$, with $\left.\cosh \xi_{0}=a / c\right)$ is a superposition of angular harmonics which vanishes at infinity $e^{-l\left(\xi-\xi_{0}\right)} \cos (l \varphi)$, and $e^{-l\left(\xi-\xi_{0}\right)} \sin (l \varphi)$. Integration of Eq. (15) across the beam boundary at $\xi=\xi_{0}$ gives rise to a jump of the derivative $\partial \Phi / \partial \xi$ that equals the surface charge on the boundary and matches the inside with the outside solution

$$
\left[\frac{\partial \Phi}{\partial \xi}\right]_{\xi_{0}-0}^{\xi_{0}+0}=\frac{\tilde{N} q^{2} / \nu_{x}^{3}}{\pi \epsilon_{0} m^{2} \gamma^{4} a^{2}}\left(e^{-2 \pi i j\left(\omega / \nu_{x}\right)}-1\right)^{-1} \int_{0}^{2 \pi j} e^{-i\left(\omega / \nu_{x}\right) u}\left[\left(p_{x}^{*} \frac{\partial \Phi}{\partial x^{*}}+T p_{y}^{*} \frac{\partial \Phi}{\partial y^{*}}\right)-v_{z} \gamma^{2}\left(p_{x}^{*} \frac{\partial A_{z}^{e}}{\partial x^{*}}+T p_{y}^{*} \frac{\partial A_{z}^{e}}{\partial y^{*}}\right)\right]_{P^{2}=0} d u
$$




\section{APPLICATION}

\section{A. Eigenmodes in ideal lattice}

In the case of vanishing errors in the lattice $\left(A_{z}^{e}=0\right)$, Eq. (17) reduces to the result in Ref. [4] for the eigen frequencies $\omega$ of oscillations of a KV beam in an ideal linear lattice. The eigenmodes are characterized by the leading power $l$ in the expansion of $\Phi$. Because of the $\delta$-function distribution, only leading terms in the $(x, y)$ expansion of $\Phi$ are needed to determine the eigenfrequency. Eigenmodes are also characterized by the symmetry with respect to the angular variable $\varphi$ where the even modes $[\cos (l \varphi)]$ are symmetric with regard to the $x$ axis and correspond to the well-known envelope perturbations. The odd or linear coupling modes lack this symmetry and reflect a skewing motion as found in linear coupling. Here we restrict ourselves to the second order even and odd modes and first review the results of Ref. [4]. Following the same notation we define $\sigma_{p}^{2}=\omega_{p}^{2} / \nu_{x}^{2}, \alpha=$ $\nu_{y} / \nu_{x}$, and $\eta=a / b, \sigma=\omega / \nu_{x}$. For the even modedenoted by the index $e$-the leading terms in the perturbed space-charge potential inside and outside are

$$
\begin{gathered}
\Phi_{2, e}^{\mathrm{int}}=a_{0} x^{2}+a_{2} y^{2}, \\
\Phi_{2, e}^{\mathrm{ext}}=\frac{a^{2} a_{0}}{2}+\frac{b^{2} a_{2}}{2}+\frac{\left(a^{2} a_{0}-b^{2} a_{2}\right) \cos 2 \varphi}{2 e^{2\left(\xi-\xi_{0}\right)}},
\end{gathered}
$$

and the dispersion relation results as

$$
\begin{aligned}
D_{2, e} \equiv & (1+\eta)^{2}+\sigma_{p}^{2}\left(\frac{1+2 \eta}{4-\sigma^{2}}+\frac{2 \eta+\eta^{2}}{4 \alpha^{2}-\sigma^{2}}\right) \\
& +\sigma_{p}^{4} \frac{2 \eta}{\left(4 \alpha^{2}-\sigma^{2}\right)\left(4-\sigma^{2}\right)}=0 .
\end{aligned}
$$

For the round isotropic beam with $\eta=1$ and $\alpha=1$, this simplifies to

$$
D_{2, e}=4+\frac{6 \sigma_{p}^{2}}{\left(4-\sigma^{2}\right)}+\frac{2 \sigma_{p}^{4}}{\left(4-\sigma^{2}\right)^{2}}=0,
$$

which is solved by the familiar result $\omega^{2}=4 \nu^{2}+\omega_{p}^{2}$ for the fast mode, and $\omega^{2}=4 \nu^{2}+\frac{\omega_{p}^{2}}{2}$ for the slow mode.

In Fig. 1 we show the ratio of envelope deviations as function of $\nu_{x} / \nu_{y}$ for the case of a homogeneous beam in a perfect lattice and with $\epsilon_{x} / \epsilon_{y}=1$ and $\Delta \nu_{y}=0.2$. Note that the incoherent space-charge tune shift is written as a positive number, hence $\nu=\nu_{0}-\Delta \nu$; also note that $\nu_{0, x} / \nu_{0, y}$ varies from 0.953 to 1.056 . We linearize the effective potential Eq. (2) and compare with coefficients of $x^{2}$ and $y^{2}$ of Eq. (18) for the beam interior, which results in a relation for the envelope ratio as function of the beam parameters and the coherent mode frequency as given by Eq. (20). The two different branches in Fig. 1 correspond to different signs of Eq. (20) for the coherent mode frequencies. Note that for the case of equal emittances as in Fig. 1 we have $\delta a / \delta b=1$ in the upper branch ("fast mode") and $\delta a / \delta b=-1$ in the lower branch

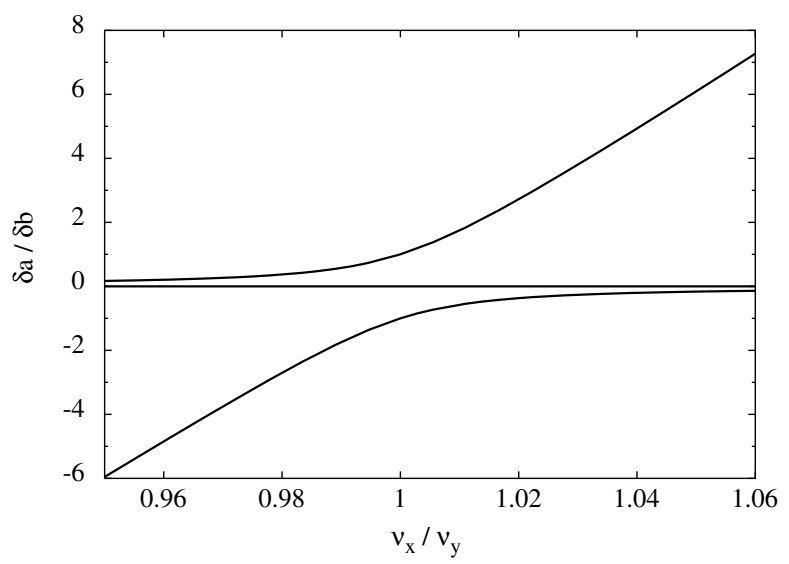

FIG. 1. Ratio of envelope deviations for the two eigenmodes of an anisotropic beam in a perfect lattice with $\epsilon_{x} / \epsilon_{y}=1$ and $\Delta \nu_{y}=0.2$.

("slow mode") for $\nu_{x} / \nu_{y}=1$. This leads to the distinction-also valid in the general anisotropic case - into a breathing (in-phase) mode for the upper branch, and a quadrupolar (out-of-phase) mode for the lower branch. For larger space-charge tune shifts the graph in Fig. 1 is stretched accordingly.

For the second order odd mode $(o)$, we assume $\Phi_{2, o}^{\mathrm{int}}=$ $a_{1} x y$ and $\Phi_{2, o}^{\text {ext }}=a b a_{1} \sin 2 \varphi / 2 e^{2\left(\xi-\xi_{0}\right)}$, which results in the dispersion relation

$$
\begin{aligned}
D_{2, o} \equiv & (1+\eta)^{2}+\frac{\sigma_{p}^{2}}{2}\left(\frac{(1-\alpha)\left(1-\eta^{2} / \alpha\right)}{(1-\alpha)^{2}-\sigma^{2}}\right. \\
& \left.+\frac{(1+\alpha)\left(1+\eta^{2} / \alpha\right)}{(1+\alpha)^{2}-\sigma^{2}}\right)=0 .
\end{aligned}
$$

For the isotropic round case this reduces to $D_{2, o} \equiv 4+$ $\left[2 \sigma_{p}^{2} /\left(4-\sigma^{2}\right)\right]=0$, with the result $\omega^{2}=4 \nu^{2}+\frac{1}{2} \omega_{p}^{2}$ which coincides with the even slow mode frequency.

\section{B. Perturbed lattice}

In the following we apply the above perturbational analysis to the inhomogeneous case in the presence of lattice errors.

\section{Envelope modes excited by gradient errors}

We first use Eq. (17) to obtain the resonance condition for the second order even mode perturbation-the envelope mode case studied by Venturini and Gluckstern [1], but generalized here to unequal focusing and emittances. The question of interest is, for instance, to see what the effect of a purely symmetric magnet perturbation and an antisymmetric perturbation is on the resonance. Note that according to [1] the symmetric perturbation (s) only excites the fast mode, whereas the antisymmetric (as) excites the slow mode only. With anisotropy this must not be the case and we expect mixing since the symmetric and antisymmetric perturbations, $x^{2}+y^{2}$ and $x^{2}-y^{2}$, 
mix. We assume

$$
\begin{aligned}
\Phi_{2, e}^{\mathrm{int}} & =\left(a_{0} x^{2}+a_{2} y^{2}\right) e^{-i\left(\omega / \nu_{x}\right) u}, \\
\Phi_{2, e}^{\mathrm{ext}} & =\left(\frac{a^{2} a_{0}}{2}+\frac{b^{2} a_{2}}{2}+\frac{\left(a^{2} a_{0}-b^{2} a_{2}\right) \cos 2 \varphi}{2 e^{2\left(\xi-\xi_{0}\right)}}\right) e^{-i\left(\omega / \nu_{x}\right) u}, \\
A_{z}^{e} & =g_{\mathrm{as}}\left(x^{2}-y^{2}\right) e^{-i\left(\omega / \nu_{x}\right) u}+g_{\mathrm{s}}\left(x^{2}+y^{2}\right) e^{-i\left(\omega / \nu_{x}\right) u},
\end{aligned}
$$

with $g$ the amplitude of the error. Different from the homogeneous case, the time dependence is now enforced by the periodic lattice error of harmonic $N$; hence, $\omega$ is replaced by $N \omega_{0}$, where $\omega_{0}$ is the angular revolution frequency. For convenience we have also introduced the scaled quantity $\sigma_{0}=\omega_{0} / \nu_{x}$. After some algebraic manipulation we obtain

$$
\begin{aligned}
& a_{0}=v_{z} \gamma^{2} \frac{\sigma_{p}^{2} \frac{2 \eta+1}{4-\left(N \sigma_{0}\right)^{2}}\left(g_{\mathrm{as}}+g_{\mathrm{s}}\right)+\sigma_{p}^{2} \frac{1}{4 \alpha^{2}-\left(N \sigma_{0}\right)^{2}}\left(g_{\mathrm{s}}-g_{\mathrm{as}}\right)+\sigma_{p}^{4} \frac{2 \eta}{\left[4 \alpha^{2}-\left(N \sigma_{0}\right)^{2}\right]\left[4-\left(N \sigma_{0}\right)^{2}\right]}\left(g_{\mathrm{as}}+g_{\mathrm{s}}\right)}{(1+\eta)^{2}+\sigma_{p}^{2}\left(\frac{1+2 \eta}{4-\left(N \sigma_{0}\right)^{2}}+\frac{2 \eta+\eta^{2}}{4 \alpha^{2}-\left(N \sigma_{0}\right)^{2}}\right)+\sigma_{p}^{4} \frac{2 \eta}{\left[4 \alpha^{2}-\left(N \sigma_{0}\right)^{2}\right]\left[\left(4-\left(N \sigma_{0}\right)^{2}\right]\right.}}, \\
& a_{2}=v_{z} \gamma^{2} \frac{\sigma_{p}^{2} \frac{\eta^{2}}{4-\left(N \sigma_{0}\right)^{2}}\left(g_{\mathrm{as}}+g_{\mathrm{s}}\right)+\sigma_{p}^{2} \frac{\eta^{2}+2 \eta}{4 \alpha^{2}-\left(N \sigma_{0}\right)^{2}}\left(g_{\mathrm{s}}-g_{\mathrm{as}}\right)+\sigma_{p}^{4} \frac{2 \eta}{\left[4 \alpha^{2}-\left(N \sigma_{0}\right)^{2}\right]\left[4-\left(N \sigma_{0}\right)^{2}\right]}\left(g_{\mathrm{s}}-g_{\mathrm{as}}\right)}{(1+\eta)^{2}+\sigma_{p}^{2}\left(\frac{1+2 \eta}{4-\left(N \sigma_{0}\right)^{2}}+\frac{2 \eta+\eta^{2}}{4 \alpha^{2}-\left(N \sigma_{0}\right)^{2}}\right)+\sigma_{p}^{4} \frac{2 \eta}{\left[4 \alpha^{2}-\left(N \sigma_{0}\right)^{2}\right]\left[4-\left(N \sigma_{0}\right)^{2}\right]}} .
\end{aligned}
$$

The resonance condition for given harmonic $N$ is given whenever the denominators vanish. From here on, unless otherwise mentioned, we drop $\omega_{0}$ by simply interpreting $\nu_{x, y}$ as tunes, i.e., betatron periods per turn. We find two solutions according to

$$
N^{2}=2 \nu_{x}^{2}+2 \nu_{y}^{2}+\left(\frac{1}{2}+\frac{a b}{(a+b)^{2}}\right) \omega_{p}^{2} \pm \frac{1}{2}\left\{\left[4\left(\nu_{x}^{2}-\nu_{y}^{2}\right)+\frac{(b-a)}{(b+a)} \omega_{p}^{2}\right]^{2}+\frac{\left(4 a^{2} b^{2}\right)}{(a+b)^{4}} \omega_{p}^{4}\right\}^{1 / 2} .
$$

For vanishing intensity this implies the incoherent conditions $2 \nu_{x}=N$ and $2 \nu_{y}=N$, while for finite intensity Eq. (26) allows one to calculate the coherently shifted tunes, which determine where the envelope resonance occurs. Further details of this relation will be discussed in Appendix A.

In Fig. 2 this resonance behavior is shown in the presence of a lattice antisymmetric error of harmonic eight from the linearization of the effective potential Eq. (2) and comparing with the second order perturbed potential and using Eqs. (24) and (25). The space charge has caused a shift from the point $\nu_{x}=4$ expected as resonance condition for the incoherent tune. Note that in this graph we have chosen fixed $\Delta \nu_{y}=0.2$; hence, $\Delta \nu_{x} \approx 0.1$ due to the given emittance ratio. The dotted line indicates the exact resonance condition at $\nu_{x}=3.96$. At this point we have $\nu_{0, x}=4.06$; hence, the self-consistent resonance allows a $60 \%$ higher space-charge tune shift as would be given by the incoherent space-charge limit $\nu_{x}=4$. This fact was also shown by Fedotov et al. [2] in the simulation study of half-integer resonance crossing in high-intensity rings. Note that in comparison with the finite envelope excursions in their simulation study we have infinite envelope response exactly at the resonance as a result of the linearized theory. Fast and slow modes are present, but since $\nu_{x}$ is bigger than $\nu_{y}$ the dominant mode is the fast one, hence $\delta a / a^{3}$ (solid lines) exceeds $\delta b / b^{3}$ (dashed lines).

\section{Odd (skew) modes excited by linear coupling}

We now use Eq. (17) to find the resonance condition for the second order odd mode. This mode causes emittance exchange in a synchrotron near a linear coupling resonance $\nu_{x}-\nu_{y}=N$, but of course modified with an additional coherent tune shift. For the odd (skew) modes we expect different shifts for the two cases $\nu_{x}+\nu_{y}+$ $\Delta \Omega_{1}=N$ (sum mode) and $\nu_{x}-\nu_{y}+\Delta \Omega_{2}=N$ (difference mode), which may be of interest in high-intensity machines with linear coupling. For the second order odd modes, we assume $[4,6]$

$$
\Phi=a_{1} x y e^{-i\left(N \omega_{0} / \nu_{x}\right) u}, \quad A_{z}^{e}=A_{1} x y e^{-i\left(N \omega_{0} / \nu_{x}\right) u},
$$

with $a_{1}$ the amplitude of the perturbed space-charge potential and $A_{1}$ the lattice error component. After some

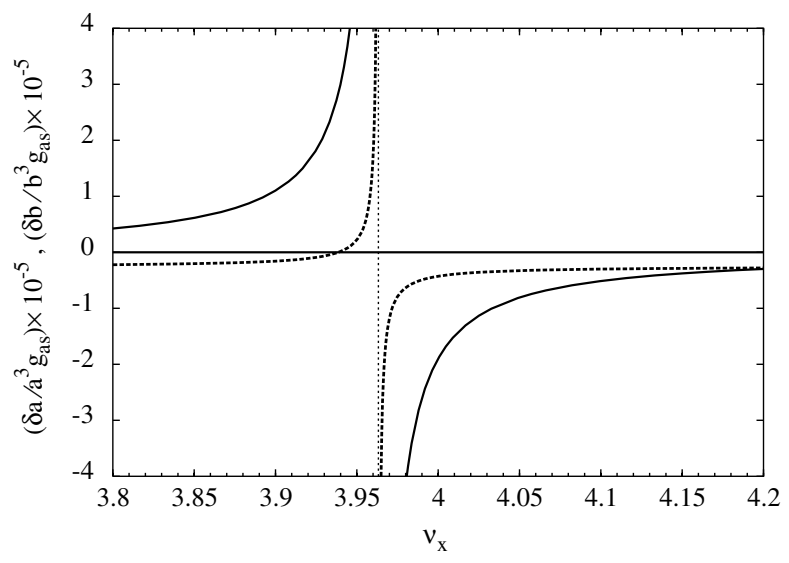

FIG. 2. Resonance behavior of the envelope deviation for an anisotropic beam with antisymmetric perturbation for $\delta a / a^{3} g_{\text {as }}$, solid lines, and $\delta b / b^{3} g_{\text {as }}$, dashed lines $\left(\epsilon_{x} / \epsilon_{y}=4\right.$, $\nu_{0, y}=3.2, N=8$, and $\Delta \nu_{y}=0.2$ for all values of $\nu_{x}$ ). 
algebra we obtain

$$
\begin{aligned}
a_{1}(1+\eta)^{2}+\left(a_{1}-A_{1} v_{z} \gamma^{2}\right)\{ & \frac{\sigma_{p}^{2}}{2}\left(\frac{(1-\alpha)\left(1-\eta^{2} / \alpha\right)}{(1-\alpha)^{2}-\left(N \sigma_{0}\right)^{2}}\right. \\
+ & \left.\left.\frac{(1+\alpha)\left(1+\eta^{2} / \alpha\right)}{(1+\alpha)^{2}-\left(N \sigma_{0}\right)^{2}}\right)\right\}=0,
\end{aligned}
$$

or

$$
a_{1}=A_{1} v_{z} \gamma^{2} \frac{\frac{\sigma_{p}^{2}}{2}\left(\frac{(1-\alpha)\left(1-\eta^{2} / \alpha\right)}{(1-\alpha)^{2}-\left(N \sigma_{0}\right)^{2}}+\frac{(1+\alpha)\left(1+\eta^{2} / \alpha\right)}{(1+\alpha)^{2}-\left(N \sigma_{0}\right)^{2}}\right)}{(1+\eta)^{2}+\frac{\sigma_{p}^{2}}{2}\left(\frac{(1-\alpha)\left(1-\eta^{2} / \alpha\right)}{(1-\alpha)^{2}-\left(N \sigma_{0}\right)^{2}}+\frac{(1+\alpha)\left(1+\eta^{2} / \alpha\right)}{(1+\alpha)^{2}-\left(N \sigma_{0}\right)^{2}}\right)},
$$

and the resonance condition reads

$$
\begin{aligned}
(1+\eta)^{2}+\frac{\sigma_{p}^{2}}{2}( & \frac{(1-\alpha)\left(1-\eta^{2} / \alpha\right)}{(1-\alpha)^{2}-\left(N \sigma_{0}\right)^{2}} \\
& \left.+\frac{(1+\alpha)\left(1+\eta^{2} / \alpha\right)}{(1+\alpha)^{2}-\left(N \sigma_{0}\right)^{2}}\right)=0
\end{aligned}
$$

which can be rewritten as

$$
\begin{aligned}
N^{2}=\left(\nu_{x}^{2}+\nu_{y}^{2}\right) & \\
+\frac{\omega_{p}^{2}}{2} \frac{a^{2}+b^{2}}{(a+b)^{2}} \pm & \frac{1}{2}\left\{\left(4 \nu_{x} \nu_{y}+\omega_{p}^{2} \frac{\left(a^{2}+b^{2}\right)}{(a+b)^{2}}\right)^{2}\right. \\
& \left.+8 \omega_{p}^{2} \frac{\left(\nu_{x}-\nu_{y}\right)\left(a^{2} \nu_{x}-b^{2} \nu_{y}\right)}{(a+b)^{2}}\right\}^{1 / 2},
\end{aligned}
$$

by returning to $\nu_{x}$ and $\nu_{y}$ as machine tunes and relating the "+" to the sum and the "-" to the difference resonance. For vanishing intensity Eq. (31) leads to the condition $\nu_{x} \pm \nu_{y}=N$ as expected. Likewise, we obtain $\nu_{x}-\nu_{y}=N$ for the difference resonance condition whenever the emittances are equal $\left(a^{2} \nu_{x}=b^{2} \nu_{y}\right)$. In Fig. 3 we plot the normalized response of the spacecharge coupling potential as function of $\nu_{x}$ for the $N=$ 1 (difference resonance) and the $N=7$ (sum resonance) cases. Because of the coherent space-charge effect the exact resonance points (dotted lines with infinite response in the linearized theory) are shifted from the expected single-particle resonance condition $\nu_{x} \pm \nu_{y}=$ $N$ (in our example $\nu_{x}=4$ ) in Fig. 3. The resonance condition for the difference mode is always found to be shifted above the single-particle resonance condition $\left(\Delta \Omega_{2}<0\right)$ as long as $\epsilon_{x} / \epsilon_{y}>1$, and down from it for $\epsilon_{x} / \epsilon_{y}<1$ (as a rule of thumb the coherent linear coupling difference resonance always occurs at increased single-particle tunes in the plane, where the emittance is larger). For the sum mode resonance we always find a shift below the single-particle resonance condition $\left(\Delta \Omega_{1}>0\right)$, which is similar to the envelope equations.

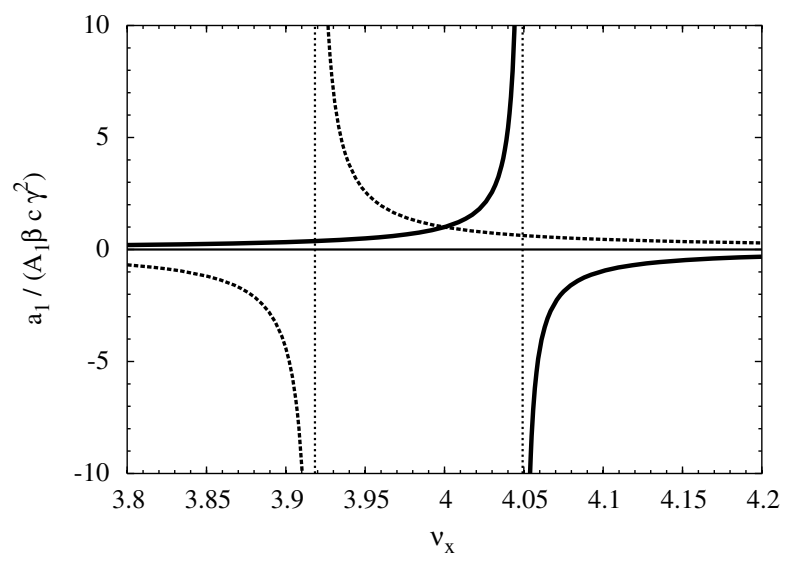

FIG. 3. Normalized response for linear coupling mode sum $(N=7$, dashed lines) and difference resonances $(N=1$, continuous lines) $\left(\epsilon_{x} / \epsilon_{y}=4, \Delta \nu_{y}=0.2, \nu_{0, y}=3.2\right)$ indicating the coherent tune shifts away from $\nu_{x}=4$.

Numerical evaluation for the difference resonance shows that the ratio of the coherent tune shift of linear coupling, normalized to the incoherent tune shift, i.e., $\left|\Delta \Omega_{2}\right| / \Delta \nu_{x}$, is mainly a function of the emittance ratio and nearly independent of the incoherent tune shift as long as the latter is small compared with the tune itself, which is generally the case in circular accelerators. In Fig. 4 we plot this dependence for $N=1$ and three different values of $\Delta \nu_{y}$ equal to $0.1,0.2$, and 0.5 . It is seen that for $0.1,0.2$ the curves practically coincide, whereas for 0.5 there is a small deviation. Although Fig. 4 was determined for $\nu_{0, y}=3.2$, cross-checks up to significantly larger tunes and $N=1,2$ have shown that the graph is practically universally applicable (with variations of at most a few percent). For the sum resonance the dependence of the thus normalized coherent tune shift is only very weak, and the corresponding graph in Fig. 4 is

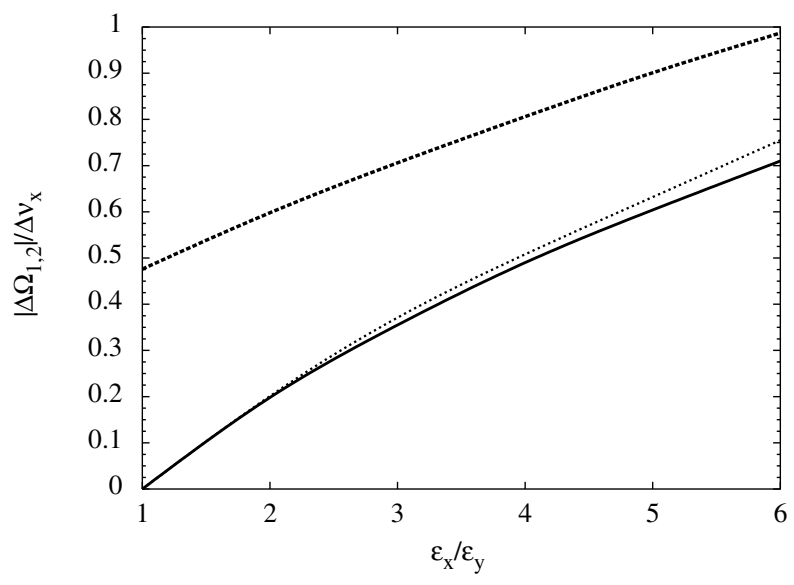

FIG. 4. Normalized coherent tune shifts $\left|\Delta \Omega_{2}\right| / \Delta \nu_{x}$ for difference resonance as function of emittance ratio for $\Delta \nu_{y}=$ $0.1,0.2$ (continuous lines coinciding) and 0.5 (dotted line). Also $\Delta \Omega_{1} / \Delta \nu_{x}$ shown for sum resonance (dashed line). 
practically also universally applicable for all values of $N$ or tunes.

A similar coherent tune shift phenomenon is obtained for tunes not split by an integer, when the coupling is driven by $N=0$ (difference resonance) as shown in Fig. 5 . In this case the exact resonance condition with infinite response (dotted line) is found to coincide - for arbitrary emittance ratio or tune - with the condition $\nu_{0, x} / \nu_{0, y}=1$ (note that this occurs in Fig. 5 at the point $\nu_{x}=3.1$, which is consistent with $\Delta \nu_{x} \approx \Delta \nu_{y} / 2$ for the emittance ratio of four). This case, however, reveals an additional feature: the odd mode is exponentially unstable in the tune range bounded by the coherent resonance condition and the single-particle resonance condition. This can be seen from the dispersion relation Eq. (30) for $N=0$, which is identical with Eq. (22) and $\sigma=\omega=0$. As was shown in Refs. [4,7] the case of zero mode frequency $\omega$ marks a transition to $\omega^{2}<0$. This implies a pair of exponentially growing $(\mathfrak{I} \omega>0)$ or damped $(\mathfrak{\Im} \omega<0)$ modes. This might be explained as a result of the destabilizing effect if the tune ratio in the interior of the beam (here $\nu_{x} / \nu_{y}>1$ ) is reversed with respect to the ratio far away from the beam, where space charge is ineffective (here $\nu_{0, x} / \nu_{0, y}<1$ ).

The growth rate of this linear coupling instability (in units of the betatron frequency in $y$, times 100) is shown as a dashed line in Fig. 5. The number of betatron periods per $e$-foldings of the growth results as $2 \pi \mathfrak{s} \omega / \nu_{0, y}$; hence, the maximum value of 0.016 in the center of the unstable region corresponds to $\approx 10$ zero space-charge betatron periods (three turns in our example). For weaker space charge also note that the growth rate is roughly proportional to the incoherent tune shift. In this instability region the exponential instability is expected to be by far dominant over the lattice error induced response. From a practical point of view the linear coupling instability band is adjacent to the "Montague resonance" stop

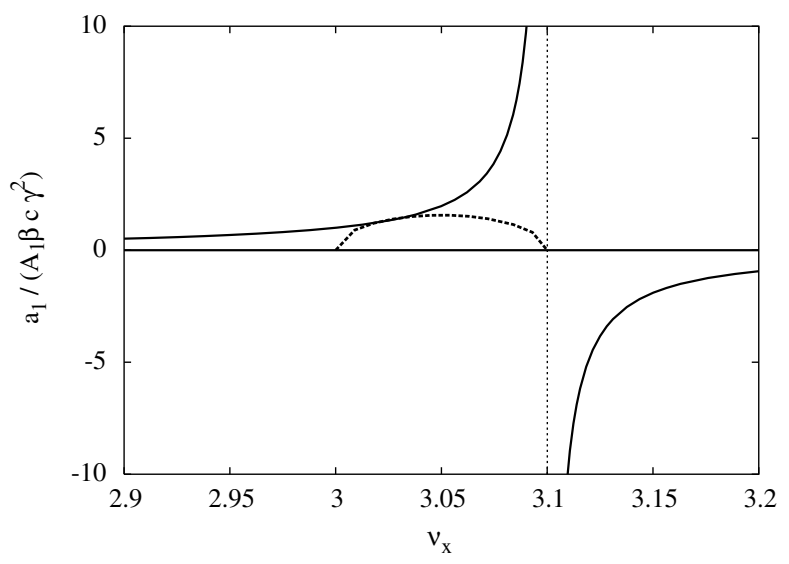

FIG. 5. Linear coupling difference resonance $(N=0)$ and linear coupling instability (dashed line is normalized instability growth rate times 100$)\left(\epsilon_{x} / \epsilon_{y}=4, \Delta \nu_{y}=0.2, \nu_{0, y}=3.2\right)$. band, which is a fourth order resonance (precisely speaking it is also an instability with exponential growth) driven by space charge for $N=0$. Detailed simulations presented in Refs. [7,8] for ideal lattices (no linear coupling errors) have shown that both processes can be identified in realistic simulations, although the fourth order resonance is found to be the dominant phenomenon in the emittance exchange.

As before in Fig. 4 it is again convenient to express results in a normalized way (see Fig. 6). We find that graphs practically coincide for the considered tune shifts up to $\Delta \nu_{y}=0.5$. This result is universally applicable to all tunes as long as $\Delta \nu_{y} \ll \nu_{y}$. Hence the presented graph allows calculation of the resonance condition of linear coupling with space charge in all cases of practical relevance.

In comparing the coherent shifts for unsplit $(N=0)$ and split tunes $(N>0)$ we note that the latter are generally about half the former for the same emittance ratios.

A practical implication of the strong dependence of the linear coupling coherent tune shift on the emittance ratio could be the following: with emittance exchange progressing it can be expected that the resonance condition is shifted away from the actual tune. Such a detuning effect might even suppress an emittance exchange if the resonance were dynamically crossed from below in the example of Fig. 3; for crossing from above the emittance exchange might be more effective, since the resonance condition moves in the same direction. Obviously, this goes beyond the linearized theory and requires computer simulation.

\section{Cancellation of lattice error by space-charge perturbation.}

The results of the preceding section can be understood more easily by exploring the relative effect of space charge on the external lattice error.

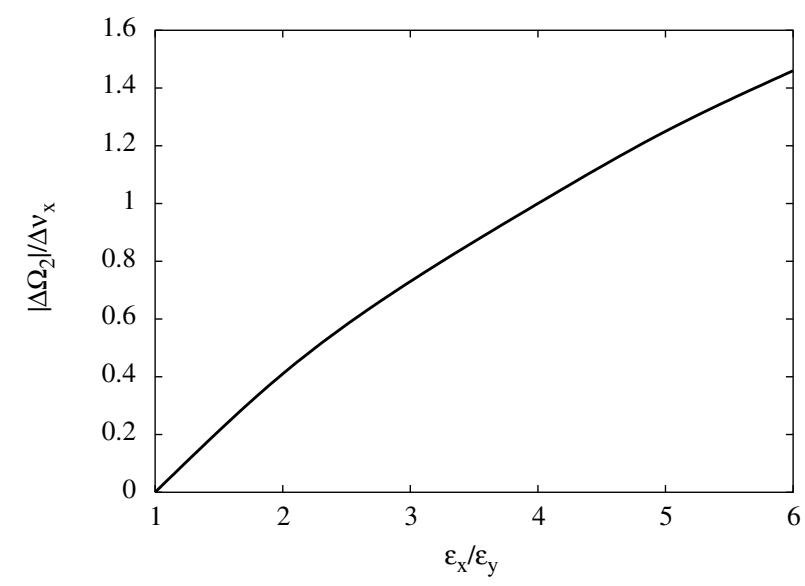

FIG. 6. Normalized coherent tune shift $\left|\Delta \Omega_{2}\right| / \Delta \nu_{x}$ for $N=0$ as function of emittance ratio. 
The total perturbation for the second order odd mode can be written as

$$
\Phi-v_{z} \gamma^{2} A_{z}^{e}=\left(a_{1}-v_{z} \gamma^{2} A_{1}\right) x y e^{-i u N \omega_{0} / \nu_{x}} .
$$

Substituting $a_{1}$ from Eq. (29) yields

$$
\Phi-v_{z} \gamma^{2} A_{z}^{e}=\frac{-A_{1} v_{z} \gamma^{2}(1+\eta)^{2}}{(1+\eta)^{2}+\frac{\sigma_{p}^{2}}{2}\left(\frac{(1-\alpha)\left(1-\eta^{2} / \alpha\right)}{(1-\alpha)^{2}-\left(N \sigma_{0}\right)^{2}}+\frac{(1+\alpha)\left(1+\eta^{2} / \alpha\right)}{(1+\alpha)^{2}-\left(N \sigma_{0}\right)^{2}}\right)} x y e^{-i u N \omega_{0} / \nu_{x}}
$$

If the denominator goes to infinity we have $(1-\alpha)^{2}-\left(N \sigma_{0}\right)^{2}=0$ and $(1+\alpha)^{2}-\left(N \sigma_{0}\right)^{2}=0$, which in turn means that $N=\nu_{x} \pm \nu_{y}$ with $\nu_{x}$ and $\nu_{y}$ the usual tunes. It is seen that the lattice error is compensated by the space-charge perturbation if the single-particle resonance condition is satisfied.

To show a similar effect for the second order even perturbations, we can write

$$
\Phi-v_{z} \gamma^{2} A_{z}^{e}=a_{0} x^{2}+a_{2} y^{2}-v_{z} \gamma^{2}\left[g_{\text {as }}\left(x^{2}-y^{2}\right)+g_{\mathrm{s}}\left(x^{2}+y^{2}\right)\right] \text {. }
$$

Substituting from Eqs. (24) and (25), this gives

$$
\begin{aligned}
\Phi-v_{z} \gamma^{2} A_{z}^{e}= & v_{z} \gamma^{2} \frac{-\left(g_{\mathrm{s}}+g_{\mathrm{as}}\right)(1+\eta)^{2}-g_{\mathrm{as}} \sigma_{p}^{2} \frac{(1+\eta)^{2}}{4 \alpha^{2}-\left(N \sigma_{0}\right)^{2}}-g_{\mathrm{s}} \sigma_{p}^{2} \frac{2 \eta+\eta^{2}-1}{4 \alpha^{2}-\left(N \sigma_{0}\right)^{2}}}{(1+\eta)^{2}+\sigma_{p}^{2}\left(\frac{1+2 \eta}{4-\left(N \sigma_{0}\right)^{2}}+\frac{2 \eta+\eta^{2}}{4 \alpha^{2}-\left(N \sigma_{0}\right)^{2}}\right)+\sigma_{p}^{4} \frac{2 \eta}{\left[4 \alpha^{2}-\left(N \sigma_{0}\right)^{2}\right]\left[4-\left(N \sigma_{0}\right)^{2}\right]}} x^{2} e^{-i u N \omega_{0} / \nu_{x}} \\
& +v_{z} \gamma^{2} \frac{-\left(g_{\mathrm{s}}-g_{\mathrm{as}}\right)(1+\eta)^{2}+g_{\mathrm{as}} \sigma_{p}^{2} \frac{(1+\eta)^{2}}{4-\left(N \sigma_{0}\right)^{2}}-g_{\mathrm{s}} \sigma_{p}^{2} \frac{2 \eta-\eta^{2}+1}{4-\left(N \sigma_{0}\right)^{2}}}{(1+\eta)^{2}+\sigma_{p}^{2}\left(\frac{1+2 \eta}{4-\left(N \sigma_{0}\right)^{2}}+\frac{2 \eta+\eta^{2}}{4 \alpha^{2}-\left(N \sigma_{0}\right)^{2}}\right)+\sigma_{p}^{4} \frac{2 \eta}{\left[4 \alpha^{2}-\left(N \sigma_{0}\right)^{2}\right]\left[4-\left(N \sigma_{0}\right)^{2}\right]}} y^{2} e^{-i u N \omega_{0} / \nu_{x}}
\end{aligned}
$$

For $4-\left(N \sigma_{0}\right)^{2}=0$, or $N= \pm 2 \nu_{x}$ using the usual tunes, the coefficient of $x^{2}$ will be equal to zero, while the $y$ coefficient remains finite. The same is true for the coefficient of $y^{2}$ if the tune has the values $N= \pm 2 \nu_{y}$ (see also Appendix B). This implies that the effect of error is compensated by the space-charge perturbation in the plane, where the single-particle resonance condition is satisfied.

\section{DISCUSSION AND CONCLUSIONS}

Our complete analysis of the linearized self-consistent response for second order resonances (the even envelope modes as well as the odd linear coupling modes) allows one to determine quantitatively the coherent spacecharge induced shift $\Omega$ in the resonance conditions $n \nu_{x}+$ $m \nu_{y}+\Delta \Omega=N(n, m=0, \pm 1$ and $N=0,1, \ldots$, while $\Omega$ depends on $n, m$, and $N$ ). The finding of exact cancellation of lattice error forces by the space-charge induced forces at the single-particle resonance condition $n \nu_{x}+$ $m \nu_{y}=N$ helps to explain this coherent shift. Asymmetry in focusing and/or emittances (beam anisotropy) are found to couple the fast and slow envelope modes in cases where the lattice gradient perturbation is purely symmetric or antisymmetric. For the linear coupling modes - the main focus of our study - we present graphs, which allow one to calculate the coherent shift $\Omega$ as a function of the emittance ratio. For weak space charge (as in rings) this coherent shift is found to be approximately independent of the space-charge tune shift as well as the absolute tune values; hence, our results allow straightforward application to determine the resonance conditions for all parameters of practical importance.
This work must be understood as a theoretical basis for future simulation and experimental studies. The strong dependence of the difference linear coupling coherent tune shift on the (unperturbed) emittance ratio suggests that the dynamical evolution, where invariant emittances only exist in a rotating frame, may be significantly affected by space charge. In cases where the linear coupling resonance is crossed for initially unequal emittances we expect an asymmetric behavior, depending on whether the crossing starts from the side to which the coherent resonance is shifted or from the opposite side. For $\epsilon_{x}>\epsilon_{y}$ the former case would imply starting the crossing from $\nu_{x} \gg \nu_{y}$; hence, the working point and coherent resonance condition move in the same direction, which might help the exchange. Obviously, a quantitative study of the effect on emittance exchange involves the nonlinear response of the linear coupling with space charge, which is beyond the present theory and will be pursued in a forthcoming study.

\section{ACKNOWLEDGMENTS}

One of the authors (M. A.) wishes to thank the Institut für Angewandte Physik at the Johann Wolfgang GoetheUniversität Frankfurt am Main for its hospitality. He also acknowledges the benefits from comments on the manuscript by J. Struckmeier.

\section{APPENDIX A}

In this paper, we obtained the tune shifts for the second order even mode based on self-consistent Vlasov-Poisson equations. A comparison with previous derivations based 
on the envelope equations is as follows:

$$
\begin{aligned}
N^{2}=2 \nu_{x}^{2} & +2 \nu_{y}^{2}+\left(\frac{1}{2}+\frac{a b}{(a+b)^{2}}\right) \omega_{p}^{2} \\
\pm & \frac{1}{2}\left\{\left[4\left(\nu_{x}^{2}-\nu_{y}^{2}\right)+\frac{(b-a)}{(b+a)} \omega_{p}^{2}\right]^{2}\right. \\
& \left.+\frac{\left(4 a^{2} b^{2}\right)}{(a+b)^{4}} \omega_{p}^{4}\right\}^{1 / 2} .
\end{aligned}
$$

Defining the following relations

$$
\begin{aligned}
& a_{0}=\frac{\omega_{p}^{2} a b}{(a+b)^{2}}=\frac{2 K}{(a+b)^{2}}, \quad a_{1}=\nu_{0, x}^{2}+\frac{3 \epsilon_{x}^{2}}{a^{4}}+a_{0}, \\
& a_{2}=\nu_{0, y}^{2}+\frac{3 \epsilon_{y}^{2}}{b^{4}}+a_{0},
\end{aligned}
$$

and using $K$ as the normalized perveance [5], we can obtain the same tune shifts as eigenvalues of the matrix

$$
A=\left(\begin{array}{cccc}
0 & 1 & 0 & 0 \\
-a_{1} & 0 & -a_{0} & 0 \\
0 & 0 & 0 & 1 \\
-a_{0} & 0 & -a_{2} & 0
\end{array}\right),
$$

which was derived by Struckmeier and Reiser [9]. Also, for the round case Eq. (A1) simplifies to

$$
N^{2}=2 \nu_{x}^{2}+2 \nu_{y}^{2}+\frac{3}{4} \omega_{p}^{2} \pm \frac{1}{2}\left\{\left[4\left(\nu_{x}^{2}-\nu_{y}^{2}\right)\right]^{2}+\frac{1}{4} \omega_{p}^{4}\right\}^{1 / 2} .
$$

Recalling that $\nu_{x}^{2}=\nu_{0, x}^{2}-\frac{\omega_{p}^{2}}{2}, \quad \nu_{y}^{2}=\nu_{0, y}^{2}-\frac{\omega_{p}^{2}}{2}$, and $\Delta \nu_{s c, x}=\frac{\omega_{p}^{2}}{4 \nu_{0, x}}$, Eq. (A4) can be rewritten as

$$
\begin{aligned}
N^{2}= & 2 \nu_{0, x}^{2}+2 \nu_{0, y}^{2}-5 \nu_{0, x} \Delta \nu_{x} \\
& \pm\left\{4\left(\nu_{0, x}^{2}-\nu_{0, y}^{2}\right)^{2}+\left(\nu_{0, x} \Delta \nu_{x}\right)^{2}\right\}^{1 / 2},
\end{aligned}
$$

which is the expression derived by Baartman [10]. Finally, for the large tune split, Eq. (A1) can be approximated for the positive sign by

$$
\begin{aligned}
N^{2}= & 4 \nu_{x}^{2}+\frac{b^{2}+2 a b}{(a+b)^{2}} \omega_{p}^{2} \\
& +\frac{a^{2} b^{2} \omega_{p}^{4}}{(a+b)^{4}\left[4\left(\nu_{x}^{2}-\nu_{y}^{2}\right)+\frac{b-a}{b+a} \omega_{p}^{2}\right]} .
\end{aligned}
$$

For the negative sign, we find

$$
\begin{aligned}
N^{2}= & 4 \nu_{y}^{2}+\frac{a^{2}+2 a b}{(a+b)^{2}} \omega_{p}^{2} \\
& -\frac{a^{2} b^{2} \omega_{p}^{4}}{(a+b)^{4}\left[4\left(\nu_{x}^{2}-\nu_{y}^{2}\right)+\frac{b-a}{b+a} \omega_{p}^{2}\right]} .
\end{aligned}
$$

From the above two expressions, we obtain the mode coefficients $C_{m}$ of Ref. [10] for the large tune split limit. Because of $\Delta \nu_{s c, x}=\omega_{p}^{2} /\left[2(1+\eta) \nu_{0, x}\right]$ and $\Delta \nu_{s c, y}=$ $\omega_{p}^{2} /\left[2(1+1 / \eta) \nu_{0, y}\right]$, we obtain

$$
C_{2 x}=\frac{3+2 \eta}{4(1+\eta)}, \quad C_{2 y}=\frac{2+3 \eta}{4(1+\eta)} .
$$

If, for example, $\eta=1$, we have $C_{2 x}=5 / 8$ and $C_{2 y}=$ $5 / 8$. For the case of $\eta=2$, this yields $C_{2 x}=7 / 12$ and $C_{2 y}=2 / 3$, in agreement with Baartman's results (see Fig. 7).

\section{APPENDIX B}

In this appendix we use the envelope equations to further illustrate the cancellation between the lattice multipole error and the induced space-charge perturbation if the single-particle resonance condition is satisfied. The beam boundary is defined by the two envelope functions $a(s)$ and $b(s)$ in the transverse plane, which satisfy the familiar nonlinear envelope equations as

$$
\begin{gathered}
\frac{d^{2}}{d s^{2}} a(s)+\kappa_{q}(s) a(s)-\frac{2 K}{a(s)+b(s)}-\frac{\epsilon_{x}^{2}}{a^{3}(s)}=0, \\
\frac{d^{2}}{d s^{2}} b(s)-\kappa_{q}(s) b(s)-\frac{2 K}{a(s)+b(s)}-\frac{\epsilon_{y}^{2}}{b^{3}(s)}=0 .
\end{gathered}
$$

In Eqs. (B1) and (B2) $\kappa_{q}(s)$ is the quadrupole focusing term. In the presence of the lattice error we write the focusing function as $\kappa_{q}\left[1+g_{m} \cos \left(\frac{\omega}{\nu_{x}} u\right)\right]$, with $g_{m}$ the amplitude of the small quadrupole error [1]. After linearization, we find

$$
\begin{aligned}
& \frac{d^{2}}{d s^{2}} \delta a+\kappa_{q} \delta a+\frac{2 K}{(a+b)^{2}}(\delta a+\delta b)+\frac{3 \epsilon_{x}^{2}}{a^{4}} \delta a \\
& \quad=-\kappa_{q} g_{m} a \cos \left(\frac{\omega}{\nu_{x}} u\right) . \\
& \frac{d^{2}}{d s^{2}} \delta b-\kappa_{q} \delta b+\frac{2 K}{(a+b)^{2}}(\delta a+\delta b)+\frac{3 \epsilon_{y}^{2}}{b^{4}} \delta b \\
& \quad=+\kappa_{q} g_{m} b \cos \left(\frac{\omega}{\nu_{x}} u\right) .
\end{aligned}
$$

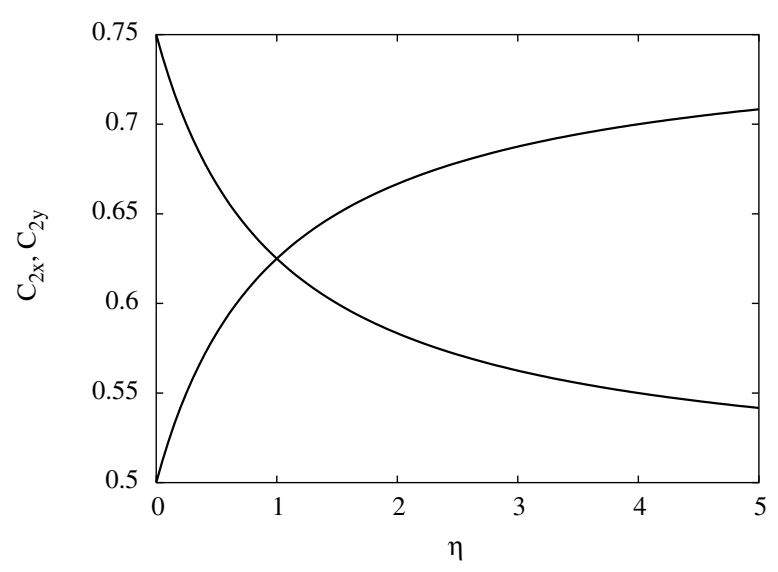

FIG. 7. Mode coefficients $C_{2 x}$ and $C_{2 y}$. 
The second order derivatives with $\beta^{2} c^{2}$ as scaled quantity

$$
\begin{aligned}
& \left(-\omega^{2}+\kappa_{q}+\frac{2 K}{(a+b)^{2}}\right) \delta a+\frac{2 K}{(a+b)^{2}} \delta b+\frac{3 \epsilon_{x}^{2}}{a^{4}} \delta a=-\kappa_{q} g_{m} a \cos \left(\frac{\omega}{\nu_{x}} u\right), \\
& \left(-\omega^{2}-\kappa_{q}+\frac{2 K}{(a+b)^{2}}\right) \delta b+\frac{2 K}{(a+b)^{2}} \delta a+\frac{3 \epsilon_{y}^{2}}{b^{4}} \delta b=+\kappa_{q} g_{m} b \cos \left(\frac{\omega}{\nu_{x}} u\right)
\end{aligned}
$$

can now be solved for $\delta a$ and $\delta b$ :

$$
\begin{gathered}
\delta a=\frac{\left[-\omega^{2}-\kappa_{q}+\frac{2 K}{(a+b)^{2}}+\frac{3 \epsilon_{y}^{2}}{b^{4}}\right] a+\frac{2 K}{(a+b)^{2}} b}{\left[\frac{2 K}{(a+b)^{2}}\right]^{2}-\left[-\omega^{2}+\kappa_{q}+\frac{2 K}{(a+b)^{2}}+\frac{3 \epsilon_{x}^{2}}{a^{4}}\right]\left[-\omega^{2}-\kappa_{q}+\frac{2 K}{(a+b)^{2}}+\frac{3 \epsilon_{y}^{2}}{b^{4}}\right]} \kappa_{q} g_{m} \cos \left(\frac{\omega}{\nu_{x}} u\right), \\
\delta b=-\frac{\left[-\omega^{2}+\kappa_{q}+\frac{2 K}{(a+b)^{2}}+\frac{3 \epsilon_{x}^{2}}{a^{4}}\right] b+\frac{2 K}{(a+b)^{2}} a}{\left[\frac{2 K}{(a+b)^{2}}\right]^{2}-\left[-\omega^{2}+\kappa_{q}+\frac{2 K}{(a+b)^{2}}+\frac{3 \epsilon_{x}^{2}}{a^{4}}\right]\left[-\omega^{2}-\kappa_{q}+\frac{2 K}{(a+b)^{2}}+\frac{3 \epsilon_{y}^{2}}{b^{4}}\right]} \kappa_{q} g_{m} \cos \left(\frac{\omega}{\nu_{x}} u\right) .
\end{gathered}
$$

For obtaining the complete resonance conditions, we have to set the denominators equal to zero. Before doing this, we cast these terms into a more familiar form. We define the following quantities:

$$
\begin{aligned}
& \epsilon_{x}=a^{2} \nu_{x}, \quad \nu_{x}^{2}=\kappa_{q}-\frac{2 K}{a(a+b)}, \quad K=\frac{\omega_{p}^{2}}{2} a b, \quad \epsilon_{y}=b^{2} \nu_{y}, \quad \nu_{y}^{2}=-\kappa_{q}-\frac{2 K}{b(a+b)}, \\
& \alpha=\frac{\nu_{y}}{\nu_{x}}, \quad \eta=\frac{a}{b}, \quad \sigma=\frac{\omega}{\nu_{x}}=\frac{N}{\nu_{x}}, \quad \sigma_{p}^{2}=\frac{\omega_{p}^{2}}{\nu_{x}^{2}} .
\end{aligned}
$$

After some algebra, the above denominators as resonance conditions become

$$
(1+\eta)^{2}+\sigma_{p}^{2}\left(\frac{1+2 \eta}{4-\sigma^{2}}+\frac{2 \eta+\eta^{2}}{4 \alpha^{2}-\sigma^{2}}\right)+\sigma_{p}^{4} \frac{2 \eta}{\left(4 \alpha^{2}-\sigma^{2}\right)\left(4-\sigma^{2}\right)}=0,
$$

which is of course consistent with the result from Eqs. (24) and (25). Now considering the motion of an individual particle, we write the equation of motion in the $x$ direction,

$$
x^{\prime \prime}+\left(\kappa_{q}-\frac{2 K}{a(a+b)}\right) x=0 .
$$

With perturbed focusing function, we have

$$
x^{\prime \prime}+\left\{\kappa_{q}\left[1+g_{m} \cos \left(\frac{N}{\nu_{x}} u\right)\right]-\frac{2 K}{a(a+b)}\right\} x=0 .
$$

We linearize the above equation,

$$
x^{\prime \prime}+\left\{\kappa_{q}\left[1+g_{m} \cos \left(\frac{N}{\nu_{x}} u\right)\right]-\frac{2 K}{(a+\delta a)(a+\delta a+b+\delta b)}\right\} x=0,
$$

yielding the following result:

$$
x^{\prime \prime}+\nu_{x}^{2} x=-\kappa_{q} g_{m} \cos \left(\frac{N}{\nu_{x}} u\right)\left\{1+\frac{2 K}{a^{2}(a+b)^{2}}[(2 a+b) \delta a+a \delta b]\right\} .
$$

From Eqs. (B7) and (B8), we substitute $\delta a$ and $\delta b$ 


$$
\begin{aligned}
x^{\prime \prime}+\nu_{x}^{2} x=-\kappa_{q} g_{m} \cos \left(\frac{N}{\nu_{x}} u\right)\{1 & +\frac{2 K}{a^{2}(a+b)^{2}}\left[\frac{(2 a+b)\left\{\left[-\omega^{2}-\kappa_{q}+\frac{2 K}{(a+b)^{2}}+\frac{3 \epsilon_{y}^{2}}{b^{4}}\right] a+\frac{2 K}{(a+b)^{2}} b\right\}}{\left[\frac{2 K}{(a+b)^{2}}\right]^{2}-\left[-\omega^{2}+\kappa_{q}+\frac{2 K}{(a+b)^{2}}+\frac{3 \epsilon_{x}^{2}}{a^{4}}\right]\left[-\omega^{2}-\kappa_{q}+\frac{2 K}{(a+b)^{2}}+\frac{3 \epsilon_{y}^{2}}{b^{4}}\right]}\right. \\
& -\frac{-a\left\{\left[-\omega^{2}+\kappa_{q}+\frac{2 K}{(a+b)^{2}}+\frac{3 \epsilon_{x}^{2}}{a^{4}}\right] b+\frac{2 K}{(a+b)^{2}} a\right\}}{\left.\left[\frac{2 K}{(a+b)^{2}}\right]^{2}-\left[-\omega^{2}+\kappa_{q}+\frac{2 K}{(a+b)^{2}}+\frac{3 \epsilon_{x}^{2}}{a^{4}}\right]\left[-\omega^{2}-\kappa_{q}+\frac{2 K}{(a+b)^{2}}+\frac{3 \epsilon_{y}^{2}}{b^{4}}\right]\right\} x .}
\end{aligned}
$$

Straightforward algebra now shows that the numerator of the above fraction vanishes for $\omega^{2}=N^{2}=4 \nu_{x}^{2}$ (not for $\omega^{2}=$ $N^{2}=4 \nu_{y}^{2}$ ); hence, there is no net perturbation of the motion of an individual particle in the $x-$ direction in this case. Repeating the above calculation similarly for $y$ gives

$$
\begin{aligned}
y^{\prime \prime}+\nu_{y}^{2} y=\kappa_{q} g_{m} \cos \left(\frac{N}{\nu_{x}} u\right)\{1 & -\frac{2 K}{b^{2}(a+b)^{2}}\left[\frac{-(a+2 b)\left\{\left[-\omega^{2}+\kappa_{q}+\frac{2 K}{(a+b)^{2}}+\frac{3 \epsilon_{x}^{2}}{a^{4}}\right] b+\frac{2 K}{(a+b)^{2}} a\right\}}{\left[\frac{2 K}{(a+b)^{2}}\right]^{2}-\left[-\omega^{2}+\kappa_{q}+\frac{2 K}{(a+b)^{2}}+\frac{3 \epsilon_{x}^{2}}{a^{4}}\right]\left[-\omega^{2}-\kappa_{q}+\frac{2 K}{(a+b)^{2}}+\frac{3 \epsilon_{y}^{2}}{b^{4}}\right]}\right. \\
& +\frac{b\left\{\left[-\omega^{2}-\kappa_{q}+\frac{2 K}{(a+b)^{2}}+\frac{3 \epsilon_{y}^{2}}{b^{4}}\right] a+\frac{2 K}{(a+b)^{2}} b\right\}}{\left.\left[\frac{2 K}{(a+b)^{2}}\right]^{2}-\left[-\omega^{2}+\kappa_{q}+\frac{2 K}{(a+b)^{2}}+\frac{3 \epsilon_{x}^{2}}{a^{4}}\right]\left[-\omega^{2}-\kappa_{q}+\frac{2 K}{(a+b)^{2}}+\frac{3 \epsilon_{y}^{2}}{b^{4}}\right]\right\} y .}
\end{aligned}
$$

Again, the right-hand side equals zero for $\omega^{2}=N^{2}=$ $4 \nu_{y}^{2}$, but not for $\omega^{2}=N^{2}=4 \nu_{x}^{2}$.

[1] M. Venturini and R. L. Gluckstern, Phys. Rev. ST Accel. Beams 3, 034203 (2000).

[2] A.V. Fedotov and I. Hofmann, Phys. Rev. ST Accel. Beams 5, 024202 (2002).

[3] A.V. Fedotov, I. Hofmann, R. L. Gluckstern, and H. Okamoto, Phys. Rev. ST Accel. Beams 6, 094201 (2003).

[4] I. Hofmann, Phys. Rev. E 57, 4713 (1998).
[5] R. C. Davidson and H. Qin, Physics of Intense Charged Particle Beams in High Energy Accelerators (World Scientific, Singapore, 2001).

[6] S.Y. Lee, Accelerator Physics (World Scientific, Singapore, 1999).

[7] I. Hofmann and O. Boine-Frankenheim, Phys. Rev. Lett. 87, 034802 (2001).

[8] I. Hofmann, G. Franchetti, O. Boine-Frankenheim, J. Qiang, and R. D. Ryne, Phys. Rev. ST Accel. Beams 6, 024202 (2003).

[9] J. Struckmeier and M. Reiser, Part. Accel. 14, 227 (1984).

[10] R. Baartman, in Workshop on Space Charge Physics in High Intensity Hadron Rings, edited by A. U. Luccio and W. T. Weng, AIP Conf. Proc. No. 448 (AIP, New York, 1998), p. 56. 